TM-1670

\title{
Some Estimation Concerning Crossing Transition of the Main Injector
}

\author{
King-Yuen Ng \\ Fermi National Accelerator Laboratory \\ P.O. Box 500 \\ Batavia, Illinois 60510
}

June 1990 
TM- 1670

MI-30

\title{
SOME ESTIMATION CONCERNING CROSSING TRANSITION OF THE MAIN INJECTOR
}

\author{
King-Yuen $\mathrm{Ng}$ \\ Fermi National Accelerator Laboratory Batavia, IL 60510, U.S.A.
}

(June, 1990) 


\section{INTRODUCTION}

We estimate some parameters pertaining to the transition crossing of the Main Injector. These include the nonadiabatic time, bunch length and bunch height at transition, the microwave growth across transition driven by a longitudinal impedance, and the parameters that govern the Umstätter's and the Johnsen's effects.

\section{BUNCH LENGTH AND HEIGHT AT TRANSITION}

At time $T_{c}$ before and after transition, the bucket changes so rapidly that the bunch is not able to follow it. We call this region the nonadiabatic region. This characteristic time is given by ${ }^{1,2}$

$$
T_{c}=\left[\left(\frac{\pi \beta_{t}^{2} \gamma_{t}^{4}}{h \omega_{0}^{2} \dot{\gamma}_{t}}\right)\left(\frac{E_{0} / e}{V_{\mathrm{rf}} \cos \phi_{0}}\right)\right]^{\frac{1}{3}},
$$

where

$$
\begin{aligned}
E_{0} & =0.93827 \mathrm{GeV}, \text { rest energy of proton, } \\
\gamma_{t} & =20.4=\left(1-\beta_{t}^{2}\right)^{-1 / 2}, \text { transition gamma, } \\
\omega_{0} & =566.78 \mathrm{kHz}, \text { angular revolution frequency, } \\
h & =588, \mathrm{rf} \mathrm{harmonic} \mathrm{number,} \\
V_{\mathrm{rf}} & =2.78 \mathrm{MV}, \mathrm{rf} \text { voltage, } \\
\phi_{0} & =37.6^{\circ} \mathrm{rf} \text { phase, }
\end{aligned}
$$

and

$$
\dot{\gamma}_{t}=\frac{\omega_{0} e V_{\mathrm{rf}} \sin \phi_{0}}{2 \pi E_{0}}=163.1 \mathrm{sec}^{-1}
$$

is the rate of change of $\gamma$ at transition. We find $T_{c}=1.96 \mathrm{~ms}$. If every particle crosses transition at exactly the same time, the evolution of the bunch can be computed easily. At transition, the bunch ellipse in the longitudinal phase space is tilted. The maximum rms bunch length (not at zero momentum offset) is given by

$$
\sigma_{\tau}=\frac{2}{3^{5 / 6} \Gamma(1 / 3)}\left(\frac{A T_{\mathrm{c}}^{2} e V_{\mathrm{r}} \dot{\gamma}_{t}}{E_{0} \beta_{t}^{2} \gamma_{t}^{4}}\right)^{\frac{1}{2}}=0.371 \mathrm{~ns},
$$

where $\Gamma(1 / 3)=2.678939$ is the gamma function and the bunch area $A=0.4 \mathrm{eV} \cdot \mathrm{sec}$ has been assumed. The maximum rms energy spread is

$$
\sigma_{E}=\frac{\Gamma(1 / 3)}{3^{2 / 3} 2 \pi}\left(\frac{A \beta_{t}^{2} \gamma_{t}^{4} E_{0}}{T_{c}^{2} \dot{\gamma}_{t}}\right)^{\frac{1}{2}},
$$

or $\sigma_{E} / E=3.45 \times 10^{-3}$. Note that here $6 \pi \sigma_{E} \sigma_{\tau}$ is not equal to the bunch area $A$ because the ellipse is tilted. 


\section{MICROWAVE GROWTH}

The growth of microwave amplitudes across transition is unavoidable because, for a certain time interval, the frequency-slip parameter $\eta=1 / \gamma_{t}^{2}-1 / \gamma^{2}$ is too small to provided enough frequency spread for Landau damping. It has been shown by Courant and Synder ${ }^{1,2}$ as well as by Herrera ${ }^{3}$ that, if one assumes $\eta / E$ to be a linear function of time in that interval, the invariant of the the longitudinal phase space can be solved analytically in terms of Bessel function and Neumann function of order $2 / 3$. A dispersion relation can be set up and the growth rate can then be solved numerically. ${ }^{4}$ If we further assume that $Z / n$ is real and the bunch is gaussian in shape, the problem can be solved approximately resulting in a handy formula. ${ }^{5}$ The total growth across transition is $\exp \left(S_{b}+S_{a}\right)$, where

$$
S_{b, a}=\left.\int \operatorname{Im} \Delta \Omega d t\right|_{\operatorname{Im}_{m} \Delta \Omega>0}
$$

represent the integrated growth rate $\operatorname{Im} \Delta \Omega$ before and after transition. The handy formula gives

$$
\frac{S_{b}}{n}=\frac{S_{\mathrm{b}}}{n}=\frac{F_{1}\left[\epsilon N(Z / n) \gamma_{t}^{2 \gamma^{2}}\left(E_{0} / \epsilon\right)^{2} \sigma_{\tau}\right.}{V_{\mathrm{rr}} \sin \phi_{0}} \frac{(A / e)^{3}}{{ }^{3}},
$$

where $N$ is the number of particle of charge $\epsilon$ per bunch, $n$ is the harmonic of the microwave frequency, and $F_{1}=8.735$ is a numerical constant. In the above, $\sigma_{\tau}$ is the rms time spread of the bunch at time $-t_{0}$ when stability is lost before transition or at time $t_{0}$ when stability is regained after transition. This time $t_{0}$ and time spread $\sigma_{\tau}$ are found to be

$$
\frac{\sigma_{\tau}}{T_{c}}= \begin{cases}\frac{2}{3_{0}^{1 / 3} \Gamma(1 / 3)}\left(\frac{A e V_{\mathrm{rf}} \omega_{0} \sin \phi_{0}}{6 \pi E_{0}^{2} \beta_{t}^{2} \gamma_{t}^{4}}\right)^{\frac{2}{2}}\left(1+0.6859 \frac{t_{0}}{T_{\mathrm{c}}}\right) & \frac{t_{0}}{T_{c}} \ll 1, \\ \left(\frac{A e V \omega_{0} \sin \phi_{0}}{6 \pi^{2} E_{0}^{2} \beta_{t}^{2} \gamma_{t}^{4}}\right)^{\frac{1}{2}}\left(\frac{t_{0}}{T_{\mathrm{c}}}\right)^{\frac{1}{4}} \frac{\left(E_{0} / \epsilon\right)^{2} \sigma_{\tau}}{(A / \epsilon)^{2}}, & \frac{t_{0}}{T_{\mathrm{c}}} \gtrsim 1 .\end{cases}
$$

Assuming that $(N Z / n) \gtrsim 10$ ohms, we obtain

$$
\begin{aligned}
t_{0} & =0.0705\left(N \frac{Z}{n}\right)^{4 / 3} \mathrm{~ms}, \\
\sigma_{\tau} & =0.176\left(N \frac{Z}{n}\right)^{1 / 3} \mathrm{~ns}, \\
\frac{S_{b, a}}{n} & =1.44 \times 10^{-7}\left(N \frac{Z}{n}\right)^{7 / 3},
\end{aligned}
$$


where $N$ is in $10^{10}$ and $Z / n$ in ohms. An illustration is given in Table I. The growth of the microwave amplitude in the last row was computed by assuming a broad band centered at $1.5 \mathrm{GHz}$ corresponding to $n=16629$. One has to bear in mind that the actual growth is usually less than indicated because nonlinear effect may come in eventually to suppress the growth rate. The growth of the microwave amplitude will dilute the bunch area and lead to a growth of the bunch area. However, the relation between the two growths is not known.

The longitudinal space-charge force will help stability before transition but help instability after transition. It leads to the shortening of $t_{0}$ before transition and lengthening of $t_{0}$ after transition. Nevertheless, its contribution will not affect the estimation in Table I by very much.

\begin{tabular}{c|c|c|c}
\hline$N \frac{Z}{n}$ & $10 \Omega$ & $20 \Omega$ & $30 \Omega$ \\
\hline$t_{0}$ & $1.52 \mathrm{~ms}$ & $3.83 \mathrm{~ms}$ & $6.57 \mathrm{~ms}$ \\
\hline$\sigma_{\tau}$ & $0.380 \mathrm{~ns}$ & $0.478 \mathrm{~ns}$ & $0.547 \mathrm{~ns}$ \\
\hline$\frac{S_{b, a}}{n}$ & $3.11 \times 10^{-5}$ & $1.57 \times 10^{-4}$ & $4.04 \times 10^{-4}$ \\
\hline$e^{S_{b}+S_{a}}$ & 2.81 & 13.5 & 820 \\
\hline
\end{tabular}

Table I: Microwave growth across transition

\section{UMSTÄTTER'S EFFECT}

The transverse space-charge force will lower the betatron tune of those particle at the transverse edge of the bunch near the center, and therefore lower the transition $\gamma$. These particles will cross transition at a time earlier than the synchronous particle. ${ }^{6}$ This effect is called the Umstätter's effect. Roughly, the depression of $\gamma_{t}$ is given by

$$
2 \gamma_{t} \Delta \gamma_{t}=-\varepsilon \lambda(0)
$$

where

$$
\lambda(0)=\frac{N}{\sqrt{2 \pi} \sigma_{\tau} h \omega_{0}}
$$


is the linear particle density at the center of the bunch and

$$
\varepsilon=\frac{4 h r_{p} R}{\beta_{t}^{2} \gamma_{t}^{3}}\left[\frac{1}{a(a+b)}-\frac{\epsilon_{1}}{h_{v}^{2}}\right] .
$$

with $a$ and $b$ the half-width and half-height of the beam, $r_{p}=1.535 \times 10^{-18} \mathrm{~m}$ the classical proton's radius, $R=528.30 \mathrm{~m}$ the ring radius, $\epsilon_{1}=0.172$ the electrostatic image coefficient corresponding to rectangular $2 "$ by 4 " beam pipe, and $h_{v}$ the halfheight of the vacuum chamber. Assuming a normalized transverse emmitance of the beam $20 \pi \mathrm{mm} \mathrm{mr}$ and a minimum beta-function $11.6 \mathrm{~m}$, we get $a=b=6.74 \mathrm{~mm}$ and therefore $\varepsilon=2.52 \times 10^{-12}$. Using $\sigma_{\tau}=0.380 \mathrm{~ns}$ and $N=5.10 \times 10^{10}$ from Section II, we get for the maximum linear density $\lambda(0)=16.0 \times 10^{10}$. This leads to a maximum depression of $\Delta \gamma_{t}=0.00995$. From Eq. (2.2), the rate of acceleration is $\dot{\gamma}_{t}=163.1$. Therefore, some particles at the center of the bunch will cross transition at a time $\Delta T=\Delta \gamma_{t} / \dot{\gamma}_{t}=0.583 \mathrm{~ms}$ earlier than the synchronous particle. Since this time is much less than the adiabatic time $T_{c}=1.96 \mathrm{~ms}$, Umstätter's effect should be negligible.

\section{JOHNSEN'S EFFECT}

Each particle inside a bunch has momentum slightly different from the synchronous momentum $p_{0}$. It travels along a different closed orbit and has a different momentum compaction factor. If the momentum deviation is $\Delta p$, its orbit length is given by

$$
L=L_{0}\left[1+\alpha_{0} \frac{\Delta p}{p_{0}}\left(1+\alpha_{1} \frac{\Delta p}{p_{0}}\right)\right],
$$

where $L_{0}$ is the synchronous orbit length and $\alpha_{0}$ is the momentum compaction factor of the synchronous particle. Obviously, this off-momentum particle will have a different $\gamma_{t}$ and crosses transition at a time $\Delta T$ earlier. It can be shown that $\Delta T$, which is also called the nonlinear time, is given $\mathrm{by}^{7}$

$$
\Delta T \approx \frac{\left(\alpha_{1}-3 / 2\right) \gamma_{t}}{\dot{\gamma}_{t}}\left(\frac{\Delta E}{E}\right)
$$

where $\Delta E / E$ is approximately equal to the relative height of the bunch if every particle crosses transition at exactly the same time (or $\alpha_{1}=-1.5$ ). From Section II, $\sigma_{E} / E=3.45 \times 10^{-3}$ at transition, we have maximum $\Delta E / E \sim 8.45 \times 10^{-3}$. This gives

$$
\left.\Delta T\right|_{\max } \sim 1.057\left(\alpha_{1}+1.5\right) \mathrm{ms} .
$$


If one assumes a perfect FODO-cell structure of phase advanced $\varphi_{c}$ with two sets of sextupoles at, respectively, the F-quads and D-quads canceling a fraction $f$ of the natural chromaticity, $\alpha_{3}$ can be derived readily to give ${ }^{8}$

$$
\alpha_{1}=\frac{1+s_{c} ! 12-f}{1-s_{c} / 12}
$$

where $s_{c}=\sin \varphi_{c} / 2$. If the sextupoles are not turned on, $f=0$. The Main Injector consists of $90^{\circ}$-cells. Therefore $\alpha_{1}=25 / 23$. Thus, we obtain maximum $\Delta T=2.7 \mathrm{~ms}$ or maximum $\Delta T / T_{c}=1.4$. the growth of bunch area due to Johnsen's effect will be appreciable. ${ }^{9}$ On the other hand, if there is a complete cancelation of chromaticity, $f=1$ and $\alpha_{1}=1 / 23$. Then, $\Delta T / T_{c}=0.84$. The growth in bunch area will be much less. The details will be discussed in a future paper.

\section{REFERENCES}

1. E.D. Courant and H.S. Synder, Annals of Physics 3, 1 (1958).

2. G.K. Green and E.D. Courant, Hanbuch der Physik, Vol. XLIV, edited by S. Flügge, p. 218 (1959).

3. J.C. Herrera, Particle Accelerators 3, 49 (1972).

4. S.Y. Lee and J.M. Wang, IEEE Transaction on Nuclear Science NS32, 2323 (1985).

5. K.Y. Ng, Fermilab Report TM-1383, 1986.

6. A. Sørenssen, Particle Accelerators 6, 141 (1975).

7. K. Johnsen, Proc. of CERN Symposium on High Energy Accelerators and Pion Physics, Genera, 1956, Vol. 1, p. 106.

8. S. Peggs, Fermilab Internal Report AP Note 90-004, 1990.

9. J. Wei, Longitudinal Dynamics of the Non-Adiabatic Regime on AlternatingGradient Synchrotrons, Thesis, State Lniversity of New York at Stony Brook, May 1990. 